\title{
Differential Open Photoacoustic Helmholtz Cell
}

\author{
Tomasz Starecki · Antonina Geras
}

Received: 17 November 2013 / Accepted: 6 February 2014 / Published online: 28 February 2014

C The Author(s) 2014. This article is published with open access at Springerlink.com

\begin{abstract}
There are very few designs of the open photoacoustic Helmholtz cells, and most of them exhibit very strong penetration of the external acoustic noise inside the cell. So far the best values of external acoustic noise suppression obtained in such cells were reported at the level of about $40 \mathrm{~dB}$ to $50 \mathrm{~dB}$. This paper presents an open photoacoustic Helmholtz cell design with a differential signal detection. Both Helmholtz resonator cavities are equipped with microphones and connected with the exterior via duct-buffer-duct structures. The length and diameter of the ducts as well as volume of the acoustic buffers are selected in such a way that the acoustic impedance of the duct-buffer-duct structure at the frequency of light modulation is relatively high. As a result, the resonance of the cell is not damped, while penetration of the external acoustic noise inside the cell is strongly reduced. Preliminary analysis predicts attenuation of the external acoustic noise at the resonance frequency of the cell with a single microphone to be at the level of at least $60 \mathrm{~dB}$. Additional rejection of the external acoustic noise can be obtained with differential detection, which simultaneously doubles the photoacoustic signal component; as in the Helmholtz resonator, pressure changes in the cavities are in counterphase.
\end{abstract}

Keywords Acoustic buffers · External acoustic noise attenuation · Helmholtz resonator $\cdot$ Open photoacoustic cell

\section{Introduction}

Photoacoustic cell designs are quite often based on Helmholtz resonators. In the simplest case such a cell consists of two cavities connected with a duct (Fig. 1). Resonance

T. Starecki $(\varangle) \cdot$ A. Geras

Institute of Electronic Systems, Warsaw University of Technology,

Nowowiejska 15/19, 00-665 Warsaw, Poland

e-mail: t.starecki@ise.pw.edu.pl 


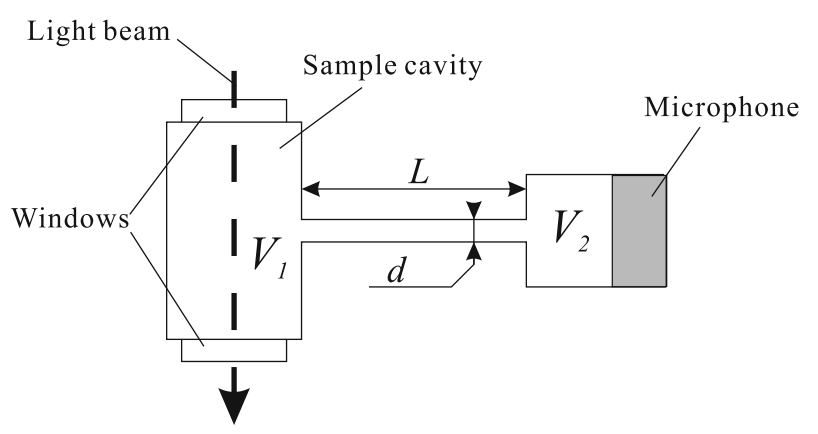

Fig. 1 Simple photoacoustic Helmholtz cell for gas measurements

of the cell can be used for amplification of the photoacoustic signal, and its frequency $f_{\text {res }}$ can be estimated as $[1,2]$

$$
f_{\mathrm{res}} \approx \frac{v d}{4 \pi} \sqrt{\frac{\pi}{L} \frac{V_{1}+V_{2}}{V_{1} V_{2}}}
$$

where $v$ is the sound velocity in the fluid inside the cell, $d$ is the duct diameter, $V_{1}, V_{2}$ are the cavity volumes, and $L$ is the duct length.

It is clearly visible from Eq. 1 that the resonance frequency of the Helmholtz cell depends, among others, on the length and diameter of the duct, which means that appropriate selection of these two dimensions allows for adjustment of the frequency of the resonance in a relatively wide range. The mentioned feature is particularly valuable in the case of low resonance frequencies (below $1 \mathrm{kHz}$ ), when the Helmholtz cell can be still kept quite compact, while dimensions of the cells based on standing wave resonators would be relatively big.

Photoacoustic Helmholtz cells are used in the investigation of all kind of samples: solids, liquids, and gases [3-7]. In the case of fluid measurements, the liquid or gas is usually supplied via an inlet and outlet on which valves are mounted [7-10]. After the cell is filled with a new sample, the valves are closed and the measurements are performed. Closing the openings (inlet and outlet) for the time of measurements has two main advantages: pressure changes resulting from the photoacoustic effect do not leak through the openings and, simultaneously, penetration of the external acoustical noise inside the cell is prevented. However, in some applications, e.g., monitoring of toxic or explosive substances, chemical reactions, etc., constant flow of the measured fluid through the cell and continuous measurements may be desirable. In such a case an open photoacoustic cell should be used.

\section{Open Photoacoustic Helmholtz Cells}

Conversion of a closed standing-wave-resonance cell into an open one can be done quite easily — by locating the inlet, outlet (and other openings, if present) in the nodes 


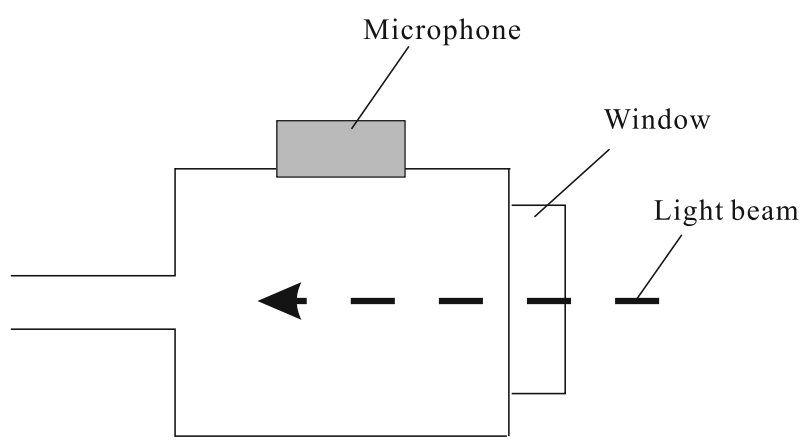

Fig. 2 Uncoupled Helmholtz resonator used as a photoacoustic cell

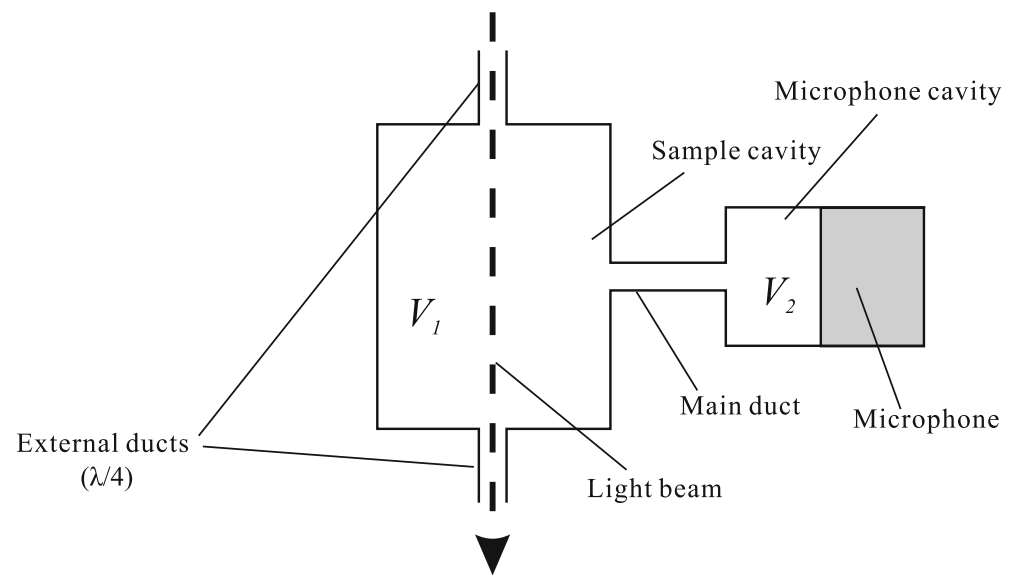

Fig. 3 Open windowless photoacoustic Helmholtz cell

of the standing wave $[11,12]$. However, in the case of a Helmholtz resonator the fluid which fills the cell is pumped between the cavities synchronously with the light stimulation without producing any standing wave. As there are no wave nodes, implementation of the opened Helmholtz cell must be based on a different mechanism. There are two possible approaches - in the first one the exterior becomes one of the two cavities forming the resonator. Such a solution was proposed by Diószeghy et al. [13], who designed the cell as an uncoupled Helmholtz resonator, opened to the ambient (Fig. 2). Unfortunately, in such a case the external acoustic noise easily propagates to the microphone cavity and is amplified by the cell resonance.

In the second approach the resonator is connected with ambient by means of acoustic components which have a relatively high acoustic impedance, so that the Helmholtz resonance is not damped. In the simplest case the mentioned components are ducts of carefully selected dimensions, connected, e.g., as in the cell presented in Fig. 3, [14]. A high acoustic impedance of the ducts can be obtained by setting their length to a quarter of the acoustic wave corresponding to the frequency of the light beam modulation. A duct of such a length should be considered as an acoustic transmission 
line. It is well known that if there is an impedance $Z_{\mathrm{o}}$ placed at the end of a quarter-wave transmission line, its input impedance $Z_{\mathrm{i}}$ equals:

$$
Z_{\mathrm{i}}=\frac{Z_{\omega}^{2}}{Z_{\mathrm{o}}}
$$

where $Z_{\omega}$ is the characteristic impedance of the line. It can be easily noticed that if $Z_{\mathrm{o}}$ is low, which is the case of a high volume acoustic cavity, then the input impedance $Z_{i}$ is high. It should be mentioned, however, that although the cell design shown in Fig. 3 will result in the resonance not being damped, it will not prevent external acoustic noise from entering the cell [15]. This results from the fact that the sample cavity has a relatively small volume; thus, a quarter-wave duct loaded with such a cavity will have a low acoustic impedance at the other end. In order to act as an efficient bidirectional acoustic separator, a quarter-wave duct must be connected to relatively large cavities at both ends. An example of such a solution is given in Fig. 4 [16], on which the external ducts are connections between exterior and acoustic buffer cavities. As both-exterior and the buffers - can be considered as large volumes; thus, the external ducts separate acoustically the main part of the cell from ambient. The internal ducts have a high acoustic impedance only from the side of the sample cavity, and their function is to connect the sample cavity with the acoustic buffers while preventing the cell resonance from being damped.

\section{Differential Open Photoacoustic Helmholtz Cell Design}

Although the cell presented in Fig. 4 had external acoustic noise attenuation at the level of $40 \mathrm{~dB}$ to $50 \mathrm{~dB}$ [17], which is substantially better in comparison to the earlier designs, it is still not enough to allow for sensitive photoacoustic measurements. It was assumed that further improvement of this parameter will be achieved by means of differential detection, which efficiently reduces the influence of the external noise. The simplest implementation of a differential detection scheme is based on two identical photoacoustic cells. One of the cells is stimulated with the light beam, while the other is used as a reference only. After subtraction of the output signals from the cells, only the photoacoustic signal component should remain, while the noise component should be substantially reduced. However, use of two cells like the one shown in Fig. 4 would result in a noticeable size increase of the whole setup (mainly due to the presence of four relatively large buffer cavities). For this reason a new cell structure as shown in Fig. 5 was designed. The main Helmholtz resonator consists of two identical cavities (Helmholtz resonator cavities) connected with a duct (main duct). Both cavities are equipped with microphones. Each of the cavities is also connected with ambient by means of an "internal duct-buffer cavity-external duct" structure, which acts as a filter reducing penetration of the external acoustic noise. The cell structure is symmetrical (plane of symmetry is perpendicular to the main duct and located at half of its length), and the openings to ambient are located on the same wall of the cell. Due to such a design, the external acoustic noise infiltrating the cell travels along identical paths (of equal acoustic impedances) inside the cell. As a result, 


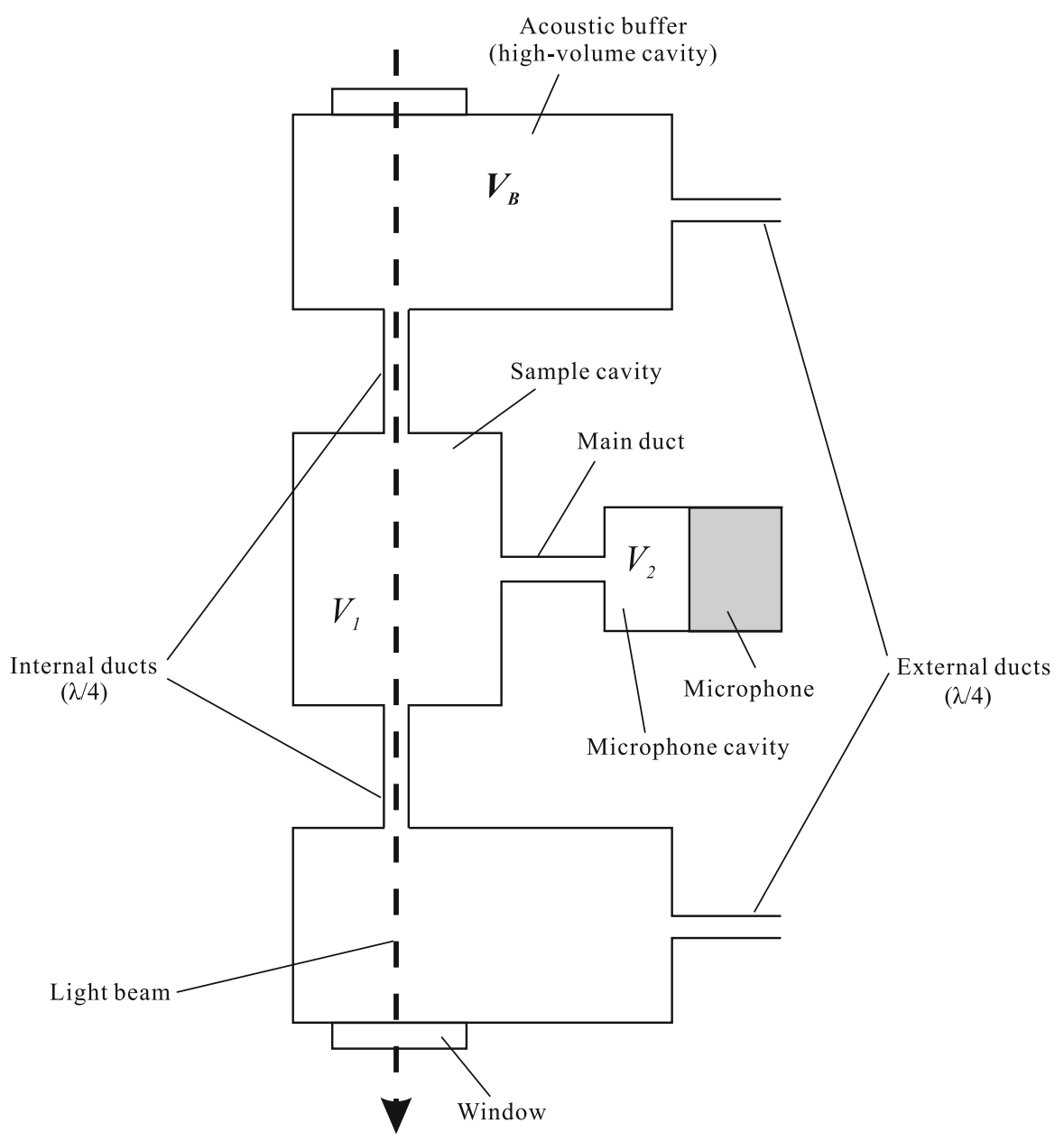

Fig. 4 Improved open photoacoustic Helmholtz cell

the residual components of the external acoustic noise (attenuated by means of the already mentioned duct-buffer-duct structures) should have very similar amplitudes and phases. Hence, taking into consideration that during differential detection the signals from the microphones located in both Helmholtz cavities are being subtracted, the level of the acoustic noise should be further decreased by a few tens of decibels. It should be noticed that during operation at the resonance frequency, photoacoustic signal components in the cavities of the Helmholtz resonator are in counterphase. Thus, subtraction of the microphone signals reduces the level of the acoustic noise, while simultaneously doubling the amplitude of the photoacoustic signal component. Additional doubling of the photoacoustic signal can be obtained by stimulating both cavities with light beams for which modulation is shifted by $180^{\circ}$ (this is schematically depicted in Fig. 5 by means of two light sources emitting light beams modulated in counterphase). 
(a)

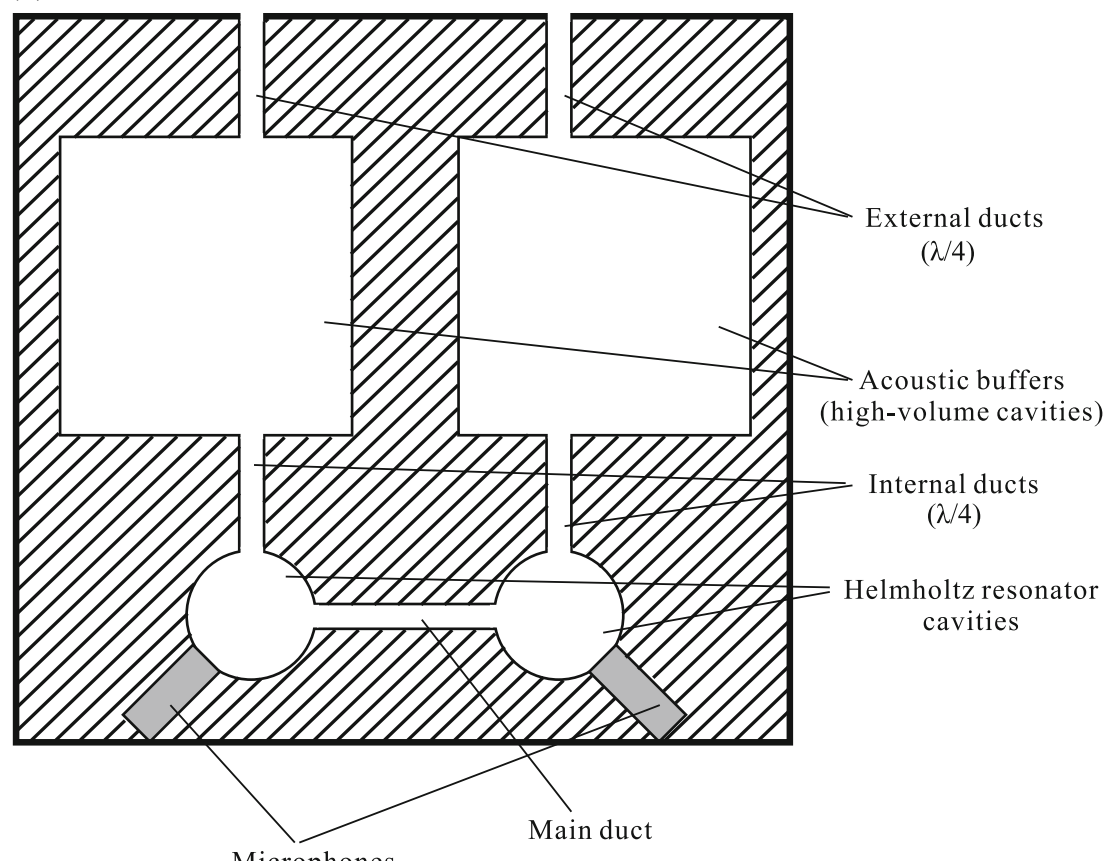

Microphones

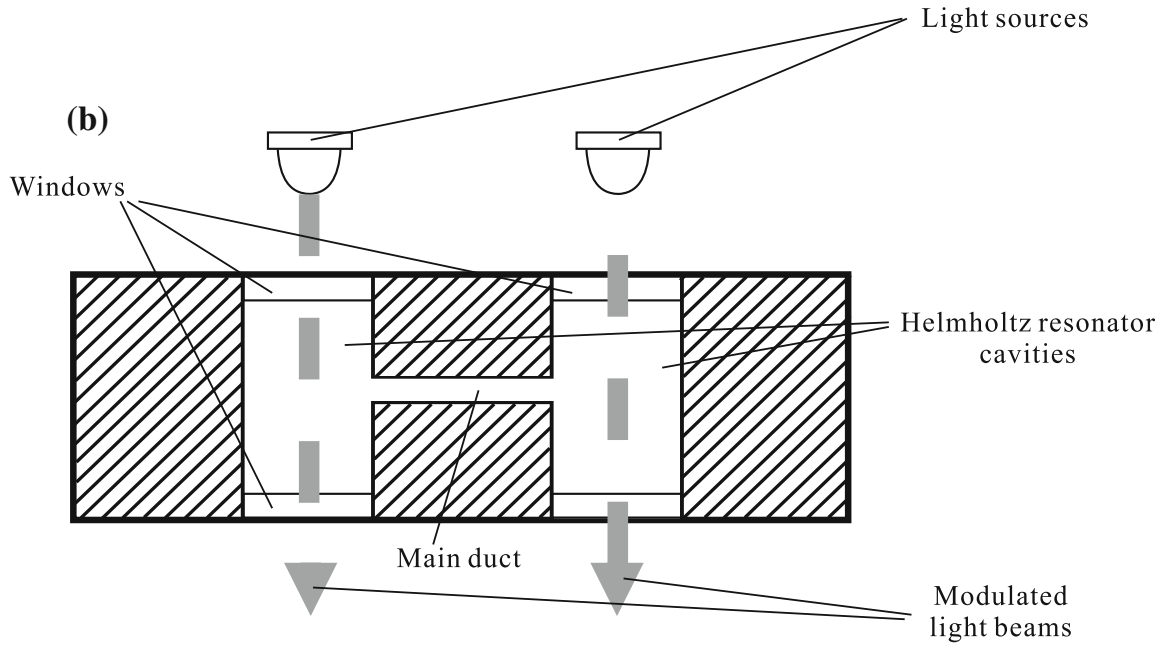

Fig. 5 Open photoacoustic Helmholtz cell with differential detection: (a) horizontal cross-section and (b) vertical cross-section

\section{Analysis of the Cell Properties}

Preliminary analysis of the properties of the presented open Helmholtz cell was done by means of computer simulations. The simulations were based on acousto-electric analog models that were previously proved to be reliable in modeling of multi-cavity 


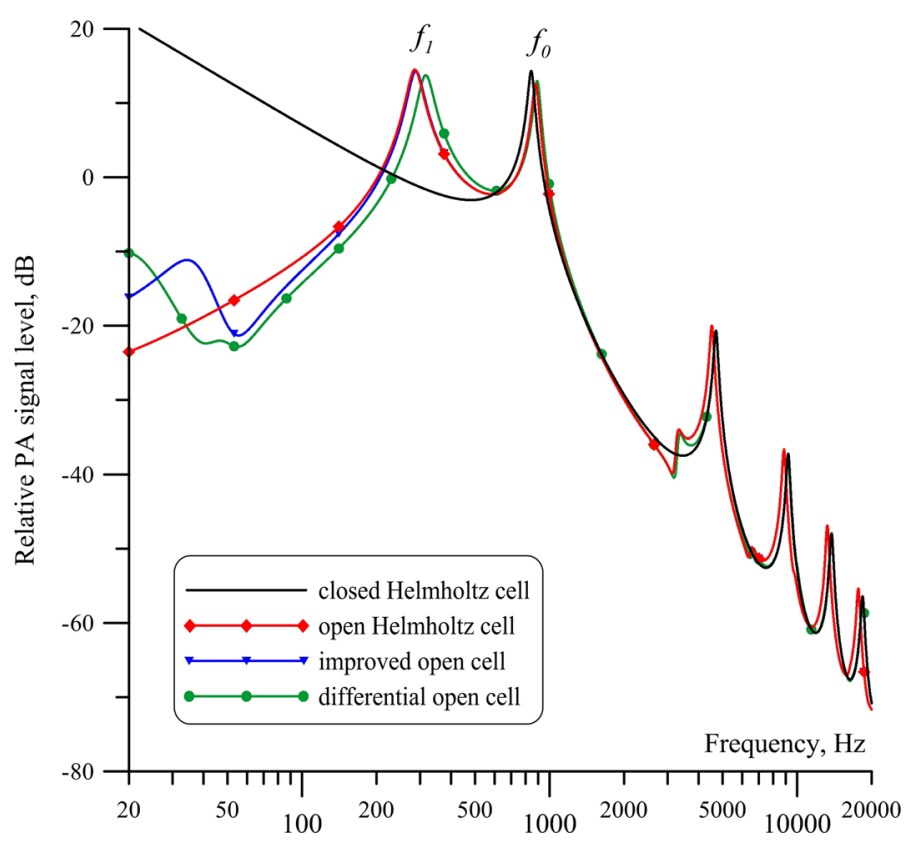

Fig. 6 Comparison of the frequency responses of the open photoacoustic Helmholtz cells

photoacoustic cell structures $[18,19]$. A more detailed description of the model used for the simulations can be found elsewhere [20]. The results were compared with the properties of previously tested open Helmholtz cells shown in Figs. 3 and 4, as well as with the properties of a closed Helmholtz cell. In order to have a similar reference point in the comparison, resonator cavities in all the cells were $1.5 \mathrm{~cm}^{3}$, while the interconnecting duct was $35 \mathrm{~mm}$ long and $3 \mathrm{~mm}$ in diameter. All the internal and external ducts had a length of $50 \mathrm{~mm}$, a diameter of $2 \mathrm{~mm}$, and the buffer volumes were $100 \mathrm{~cm}^{3}$ each. Comparison of the frequency responses of the cells is presented in Fig. 6. In the case of the differential cell, the frequency response taken for the comparison was the one showing the photoacoustic signal in one of the Helmholtz cavities while light stimulation was applied to the other cavity. It is clearly visible that at the frequencies around $f_{0}$ resonance, all the responses shown in Fig. 6 are very similar. This in particular means that both-resonance frequency and Q-factor of all the open cells - are close to the values of the properties found in the closed Helmholtz cell.

Frequency responses that show differential operation of the analyzed cell are presented in Fig. 7. The curves show the level of the photoacoustic signal in both cavities (thin lines) and the resulting differential signal (thick line) with the optical stimulation applied to only one of the Helmholtz cavities. It can be noticed that the resonance observed at the frequency $f_{1}$ at non-differential operation of the cell, is attenuated by approximately $30 \mathrm{~dB}$ at differential operation while the $f_{0}$ resonance is then twice stronger. This results from the fact that, as already mentioned, at the resonance frequency $f_{0}$ photoacoustic signal components in the Helmholtz resonator cavities are in 


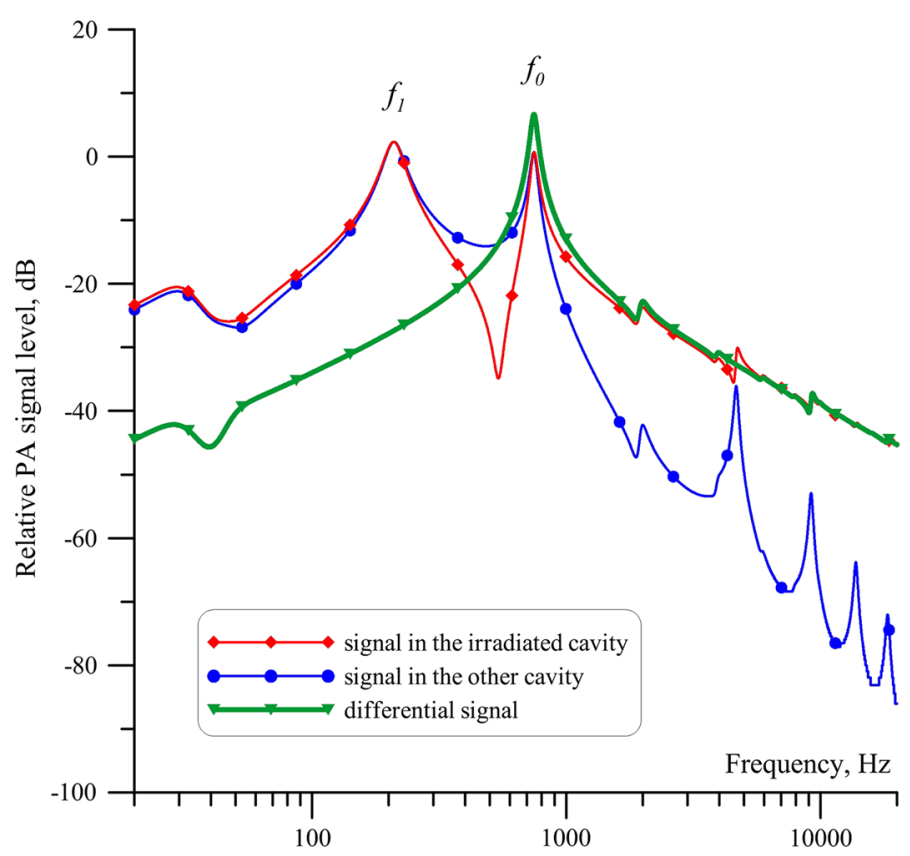

Fig. 7 Frequency responses at differential operation of the cell

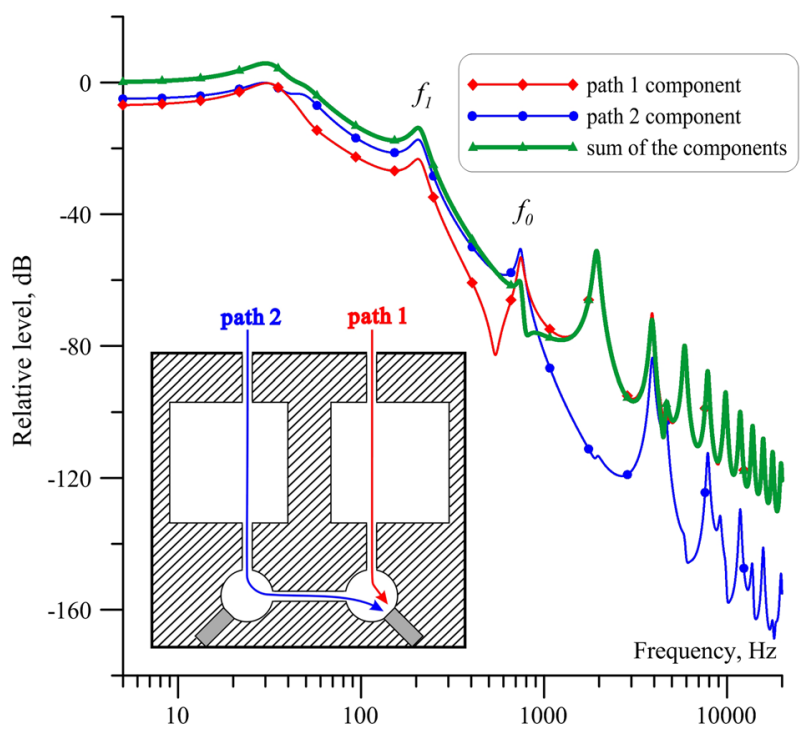

Fig. 8 External acoustic noise infiltration in the new cell (measured with a single microphone)

the counterphase, while at $f_{1}$ (the resonance resulting from interaction of the main cell with the acoustic buffers) the phase shift between the signals from the microphones is relatively small and their subtraction substantially reduces the final amplitude. 


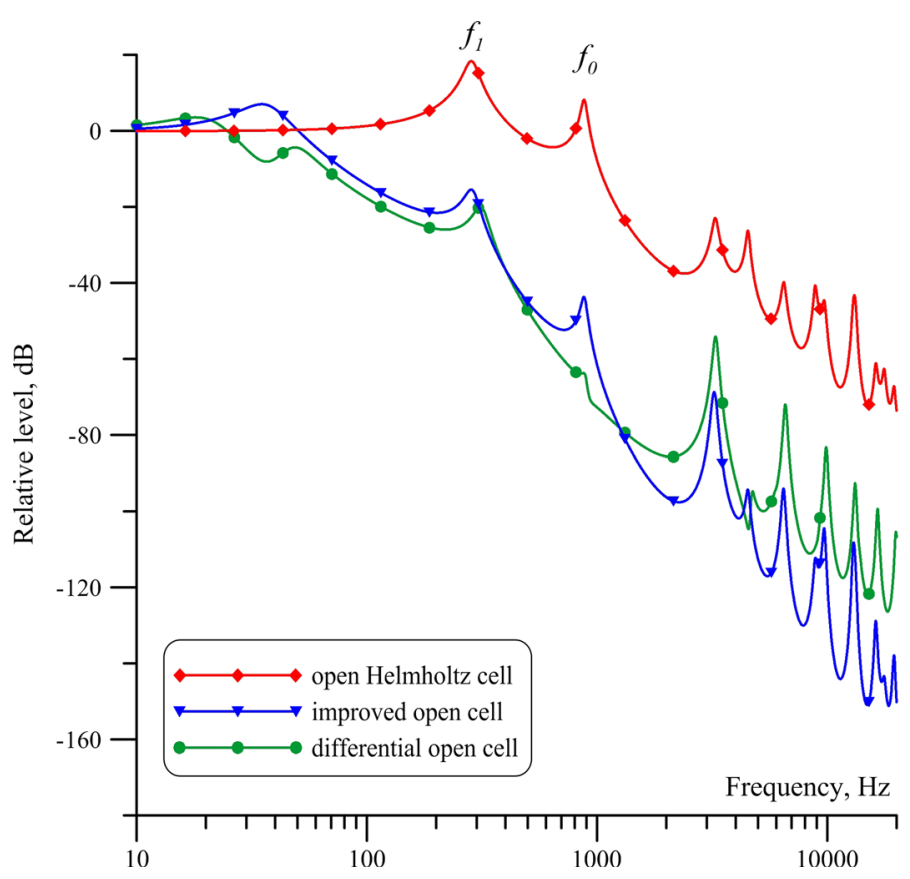

Fig. 9 Comparison of the external acoustic noise infiltration in the open photoacoustic Helmholtz cells

Although differential detection can significantly reduce influence of the external acoustic noise, it should not be the only means of the noise suppression. Inherent acoustic noise attenuation properties of the cell are presented in Fig. 8. These properties were estimated under the assumption that the amplitude and phase of the external acoustic disturbance is identical at both cell openings. Such a noise will reach a microphone located in one of the Helmholtz cavities traveling along two paths as shown in Fig. 8. It should be noticed that, at the frequency $f_{0}$, the phase of the signal inverts when the signal is traveling from one Helmholtz cavity to the other. That is why the sum of the components around $f_{0}$ is noticeably lower than for each of the single-path signal components. Finally, it can be seen that attenuation of the external noise at the $f_{0}$ resonance frequency is at the level of $60 \mathrm{~dB}$, which is a substantial improvement over previous open Helmholtz cell designs (Fig. 9). It should be also emphasized that the mentioned value describes the inherent acoustic noise attenuation properties of the cell before dimensional optimization and that such optimization and use of differential detection should still increase rejection of the noise.

\section{Conclusions}

The presented new design of an open photoacoustic Helmholtz cell preserves main frequency response properties (resonance frequency and Q-factor values) that can be found in the earlier designs of such cells. At the same time, the developed cell structure shows much higher suppression of the external acoustic noise, due to better inherent 
noise attenuation properties and differential photoacoustic signal detection. An important advantage of the applied differential detection is doubling of the photoacoustic signal amplitude. Additional increase of the photoacoustic signal can be obtained by stimulating both cavities with the light beams modulated in counterphase.

Open Access This article is distributed under the terms of the Creative Commons Attribution License which permits any use, distribution, and reproduction in any medium, provided the original author(s) and the source are credited.

\section{References}

1. A. Miklós, P. Hess, Z. Bozóki, Rev. Sci. Instrum. 72, 1937 (2001)

2. N.C. Fernelius, Appl. Opt. 18, 1784 (1979)

3. J. Bodzenta, B.D. Hanh, A. Kaźmierczak, R.H.H. Neubert, S. Wartewig, Instrum. Sci. Technol. 34, 107 (2006)

4. M.D. Rabasović, M.G. Nikolić, M.D. Dramićanin, M. Franko, D.D. Markushev, Meas. Sci. Technol. 20, 095902 (2009)

5. S.M. Park, M.I. Khan, H.Z. Cheng, G.J. Diebold, Ultrasonics 29, 63 (1991)

6. C. Lou, D. Xing, Appl. Phys. Lett. 96, 211102 (2010)

7. J. Pelzl, K. Klein, O. Nordhaus, Appl. Opt. 21, 94 (1982)

8. R. Kästle, M.W. Sigrist, Appl. Phys. B 63, 389 (1996)

9. V. Zeninari, V.A. Kapitanov, D. Courtois, YuN Ponomarev, Infrared Phys. Technol. 40, 1 (1999)

10. M.B. Pushkarsky, M.E. Webber, C.K.N. Patel, Appl. Phys. B 77, 381 (2003)

11. S. Schäfer, M. Mashni, J. Sneider, A. Miklós, P. Hess, H. Pitz, K.-U. Pleban, V. Ebert, Appl. Phys. B 66, 511 (1998)

12. S. Schilt, J.-P. Besson, L. Thévenaz, Appl. Phys. B 82, 319 (2006)

13. T. Diószeghy, A. Miklós, A. Keleman, A. Lórincz, J. Appl. Phys. 56, 2105 (1985)

14. T. Starecki, Acta Phys. Pol. A 114, A211 (2008)

15. T. Starecki, Acta Phys. Pol. A 114, A199 (2008)

16. T. Starecki, A. Geras, Int. J. Thermophys. (2013). doi:10.1007/s10765-013-1479-y

17. A. Geras, T. Starecki, Int. J. Thermophys. (2013). doi:10.1007/s10765-013-1497-9

18. T. Starecki, Proc. SPIE 6159, 61592M (2005)

19. T. Starecki, J. Acoust. Soc. Am. 122, 2118 (2007)

20. A. Geras, T. Starecki, Parametric analysis of a differential photoacoustic Helmholtz cell, Int. J. Thermophys. (2013) (submitted) 